\title{
The prognostic link between metabolic syndrome and the different breast cancer molecular subtypes: a case control study.
}

\section{Yi zuo Chen}

Wenzhou Medical University First Affiliated Hospital

\section{Yu ying Zhou}

Wenzhou Medical University First Affiliated Hospital

En dong Chen

Wenzhou Medical University First Affiliated Hospital

Di shuang Hu

Wenzhou Medical University First Affiliated Hospital

Xuan Shao

Wenzhou Medical University Second Affiliated Hospital

Ye Feng Cai ( $\nabla$ cyfoncology@gmail.com )

Wenzhou Medical University First Affiliated Hospital

Jie You ( $\nabla$ wzyoujie@163.com )

Wenzhou Medical University First Affiliated Hospital

Research

Keywords: Metabolic syndrome, Breast cancer, Molecular subtype, Prognosis

Posted Date: July 30th, 2020

DOl: https://doi.org/10.21203/rs.3.rs-42718/v1

License: (c) (i) This work is licensed under a Creative Commons Attribution 4.0 International License.

Read Full License 


\section{Title page}

\section{Title}

The prognostic link between metabolic syndrome and the different breast cancer molecular subtypes: a case control study

\section{Authors' name}

Yizuo Chen ${ }^{1 *}$, Yuying Zhou ${ }^{1 *}$, Endong $\mathrm{Chen}^{1^{*}}$, Dishuang $\mathrm{Hu}^{2}$, Xuan Shao ${ }^{3}$, Yefeng $\mathrm{Cai}^{1 *}, \mathrm{Jie} \mathrm{You}^{1 *}$

${ }^{1}$ Department of Breast \& Thyroid Surgery, the First Affiliated Hospital of Wenzhou Medical University, Wenzhou, China

${ }^{2}$ Department of Gastroenterology, the First Affiliated Hospital of Wenzhou Medical University, Wenzhou, China

${ }^{3}$ Department of breast surgery, the Second Affiliated Hospital of Zhejiang University school of medicine, Hangzhou, China

*Co-first author: Yizuo Chen, Yuying Zhou, and Endong Chen

*Co-Correspondence: Yefeng Cai and Jie You

Jie You, Department of Breast \& Thyroid Surgery, the First Affiliated Hospital of

Wenzhou Medical University, Wenzhou, Zhejiang, China.

E-mail:wzyoujie@163.com

Yefeng Cai, Department of Breast \& Thyroid Surgery, the First Affiliated Hospital of Wenzhou Medical University, Wenzhou, Zhejiang, China.

E-mail: cyfoncology@gmail.com 


\begin{abstract}
Background Metabolic syndrome (MetS) is an important factor related to the poor prognosis of breast cancer $(\mathrm{BC})$. Molecular heterogeneity in the tumor may affect the consequence of $\mathrm{BC}$. The main purpose of this study was to assess the prognostic link between MetS and the different BC molecular subtypes.
\end{abstract}

Methods A total of 960 patients with BC were recruited from January 2010 to June 2014. The relationship between MetS and disease prognosis was assessed by using univariate and multivariate analyses.

Results At recruitment, MetS was diagnosed in 199 patients (20.7\%). The mean follow-up period was 68.5 months (range, 2-103 months). MetS remained significantly associated with $64 \%$ increased risk of recurrence (Hazard Ratio $(\mathrm{HR})=1.64 ; 95 \%$ confidence interval $(\mathrm{CI}) 1.19-2.27, P<0.01)$ and twofold increased risk of mortality $(\mathrm{HR}=2.02,95 \% \mathrm{CI} 1.29-3.16, P<0.01)$ according to multivariate analysis. By reviewing BC molecular subtypes, the significant associations remained in the subsets of Luminal A ( $\mathrm{HR}=3.1,95 \%$ CI $1.28-4.57, P=0.01)$, Luminal B (Her2negative) $(\mathrm{HR}=3.3,95 \% \mathrm{CI} 1.31-8.33, P=0.01)$, and Her-2-positive (non-Luminal) $(\mathrm{HR}=2.2,95 \%$ CI $1.10-4.39, P=0.03)$.

Conclusions MetS was significantly related to unfavorable prognosis in operable BC, especially in the subgroup of Luminal A. More studies are needed to definitively determine which factors influence the association of MetS with Luminal A subtype. Keywords Metabolic syndrome; Breast cancer; Molecular subtype; Prognosis 


\section{Background}

The new breast cancer (BC) cases in the world are estimated to be more than 2 million in 2018 based on the GLOBOCAN (1). In Asia, the incidence of BC has been rising rapidly compared with the stable or decreasing incidence in western countries $(2,3)$. In China, the five-year survival rate of $\mathrm{BC}$ has increased up to $73.1 \%-83.0 \%$ in the past 15 years (4). However, according to the latest data of CONCORD, the 5-year overall survival rate of $\mathrm{BC}$ has reached $90.2 \%$ in the United States from 2010 to 2014 , whereas the 5-year overall survival rate during the same period in China is only $83.2 \%$ (5). The major differences in mortality reflected the variation in the level of knowledge regarding the importance of keeping a healthy lifestyle and the monitoring of post-treatment symptoms (6). Adopting the westernized lifestyle is considered as the main driving force of this trend in the post-treatment status of patients with $\mathrm{BC}$, which remains a controversial issue.

Metabolic syndrome (MetS) is a set of metabolic abnormalities that include hypertension, obesity, dyslipidemia, and hyperglycemia (7). Growing epidemiological data revealed that the increase in incidence and mortality of $\mathrm{BC}$ was associated with MetS $(8,9)$. Epidemiological studies have indicated the close association between MetS and increased risk of recurrence and overall mortality due to $\mathrm{BC}(7,9,10)$. Based on the intrinsic molecular subtypes, some researchers reported that a non-significant trend for increased risk of mortality was observed among the hormone-receptor (HR)positive patients with $\mathrm{BC}$ and MetS $(11,12)$. Moreover, the lack of data on Her-2 status may affect the conclusion of the impression of Mets on the prognosis of early 
$\mathrm{BC}$, which is based on the molecular profiles. Accordingly, several related studies have shown conflicting results because of the hypothesis that the different components of MetS may be involved in the development and prognosis of the molecular subtypes of BC (11-14).

This study aimed to evaluate whether MetS could affect the different prognoses of $\mathrm{BC}$ with different molecular subtypes.

\section{Methods}

\section{Study design}

The study included 960 patients with BC with operable stages I-III. We collected all clinical data from a single center, The First Affiliated Hospital of Wenzhou Medical University, between January 2010 and June 2014. Eligible patients met the following criteria: (1) women, (2) adults (>18 years old), and (3) patients without a history of other cancers. This study was approved by the Ethics Committee of the First Affiliated Hospital of Wenzhou Medical University. Written informed consent was required for all patients included in the study.

\section{Diagnostic criteria of MetS}

In this report, we applied the criteria of the Chinese Medical Association Diabetes Society (CMADS) in 2004 (15). The definition of MetS should meet at least three of following: (1) overweight and/or obese with a body mass index (BMI) of $\geq 25.0$ $\mathrm{kg} / \mathrm{m}^{2}$; (2) systolic/diastolic blood pressure of $\geq 140 / 90 \mathrm{mmHg}$ or taking drug for 
diagnosed hypertension; (3) fasting serum triglyceride level of $\geq 1.7 \mathrm{mmol} / \mathrm{L}$ $(150 \mathrm{mg} / \mathrm{dL})$ and/or high-density lipoprotein cholesterol content of $<0.9 \mathrm{mmol} / \mathrm{L}$ $(35 \mathrm{mg} / \mathrm{dL})(\mathrm{male})$ and $<1.0 \mathrm{mmol} / \mathrm{L}(39 \mathrm{mg} / \mathrm{dL})$ (female); and (4) fasting plasma glucose level of $\geq 6.1 \mathrm{mmol} / \mathrm{L}(110 \mathrm{mg} / \mathrm{dL})$ and/or $2 \mathrm{~h}$ postprandial glucose level of $\geq$ $7.8 \mathrm{mmol} / \mathrm{L}(140 \mathrm{mg} / \mathrm{dL})$ or taking drug for diagnosed diabetes mellitus.

\section{Clinical-pathological and laboratory data}

Adjuvant treatment after curative surgical resection is adjusted to the National Comprehensive Cancer Network guidelines whether or not the patient received such treatment. The pathological stage for each patient with $\mathrm{BC}$ was established according to AJCC ( $7^{\text {th }}$ edition) (16). BC molecular subtypes were classified by estrogen receptor (ER), progestogen receptor (PR), Her-2, and Ki-67 status based on the 2013

St. Gallen Consensus Statement as follows (17): Luminal A, ER-positive and PRpositive (PR $>20 \%$ ), Her2-negative, Ki-67 <20\%; Luminal B (Her-2 negative), ERpositive and/or PR-positive (PR $<20 \%$ ), Her2-negative, Ki-67 $\geq 20 \%$, or PR-negative; Luminal B (HER2-positive), ER- and/or PR-positive, HER2-positive, any Ki-67; HER2-positive (non-luminal): ER- and PR-negative, HER2-positive, any Ki-67; and triple-negative breast cancer (TNBC), ER- and PR-negative, HER2-negative, and Ki67.

\section{Follow-up evaluation}

Patients received routine follow-up evaluation after being discharged from the 
hospital and underwent a postoperative examination that included mammography, chest $\mathrm{x}$-ray, ultrasound imaging, computed tomography, and tumor biomarkers. Information about adverse events, including relapse and death, was obtained from the inpatient and outpatient records. The follow-up deadline was September 2018. Overall survival (OS) was well-defined as the period from the time of surgery to the time of demise or last follow-up. Disease-free survival (DFS) is described as the period from the time of surgery to the time of relapse or recurrence of cancer, as confirmed by radiological or histological examination, or to the last follow-up.

\section{Statistical analysis}

Differences in the clinicopathological parameters were compared between the patients with and without MetS. The comparisons were made by using Chi-square test and Student's $t$-test as appropriate. The survival curves were plotted by the Kaplan-Meier method and the differences evaluated by the log-rank test. Hazard ratio (HR) with $95 \%$ confidence interval $(\mathrm{CI})$ were estimated to express the risk effect size. When the $P$-value of the variable was less than 0.1 in univariate analysis, the variable entered the multivariate logistic regression analysis model. Entirely statistical tests were 2sided, and the correlation of statistical significance was set at 0.05 level. All analyses were conducted by using the SPSS 19.0 for Windows.

\section{Results}

Among the 960 Patients with BC, 199 patients (20.7\%) had MetS according to the 
CMADS criteria (15). The mean age was 51.8 years old. At initial diagnosis, a total of $342(35.6 \%)$ patients with BC were at stage I, 511 (53.2\%) were at stage II, and 107 $(11.1 \%)$ were at stage III. MetS is more common among the elderly $(P<0.01)$ and post-menopausal patients $(P<0.01)$. Patients with and without MetS did not differ significantly in TNM staging, tumor differentiation, molecular subtype, and therapeutic regimen (Table1).

During the mean follow-up period of 68.5 months (range, 2-103 months), a total of 120 patients died, 40 of which had MetS. According to the Kaplan-Meier survival function analysis, the patients with MetS had lower DFS $(P<0.001)$ and lower OS $(P$ $<0.001)$ than those without MetS (Figures 1A and 1B, respectively).

Based on the univariate analysis (Table 2), MetS was related to $68 \%$ greater risk of recurrence $(\mathrm{HR}=1.68 ; 95 \% \mathrm{CI} 1.21-2.32)$ and twofold greater risk of mortality $(\mathrm{HR}=2.05 ; 95 \%$ CI 1.40-2.99). In the multivariable-adjusted models, the results of recurrence and mortality were still highly consistent with the above data $(\mathrm{HR}=1.64$; 95\% CI 1.19-2.27 and HR=2.02; 95\% CI 1.29-3.16, respectively). Nevertheless, any component of MetS was not an independent risk factor for the prognosis.

In the sub-group analysis (Table 3), MetS was related to a twofold greater risk of $\mathrm{BC}$ recurrence between pre-menopausal patients $(\mathrm{HR}=2.0995 \% \mathrm{CI} 1.13-3.88$, $P=0.02$ ) and $50 \%$ higher risk of $\mathrm{BC}$ recurrence among post-menopausal women $(\mathrm{HR}=1.5495 \% \mathrm{CI} 1.04-2.30, P=0.03)$. Similar results were shown in the analysis of mortality stratified by menopausal status. In patients with normal BMI $\left(\leq 25 \mathrm{~kg} / \mathrm{m}^{2}\right)$, MetS was associated with a 2.89 -fold $(\mathrm{HR}=2.8995 \%$ CI $1.95-4.28, P<0.01)$ and 
3.58-fold $(\mathrm{HR}=3.5895 \% \mathrm{CI} 2.27-5.64, P<0.01)$ greater risk of $\mathrm{BC}$ mortality and recurrence, respectively. However, high BMI was not related to a greater risk of BC recurrence and mortality. Compared with stages II and III, MetS in stage I significantly affected both DFS and OS in early BC. In models evaluating differences by molecular subtypes, MetS was significantly related to a greater risk of BC mortality for Luminal A (HR=3.10 95\% CI 1.36-7.08, $P=0.01$ ), Luminal B (Her2negative) $(\mathrm{HR}=3.3195 \% \mathrm{CI} 1.31-8.34, P=0.01)$, and Her2-positive (non-luminal) $(\mathrm{HR}=2.1995 \%$ CI 1.10-4.39, $P=0.03)$. Additionally, MetS was significantly associated with a 2.4-fold higher risk of BC recurrence in the Luminal A group $(\mathrm{HR}=2.4295 \%$ CI $1.28-4.57, P=0.01)$.

In multivariate analysis adjusting for TNM stage, menopause status, and BMI (Table 4), MetS was still statistically significantly related to a greater risk of BC mortality for Luminal A subtype (HR=4.70, 95\% CI 1.80-2.30), Luminal B (Her2negative) $(\mathrm{HR}=3.11,95 \% \mathrm{CI} 1.10-8.77)$, and Her2-positive (non-luminal) $(\mathrm{HR}=2.85$ 95\% CI 1.18-6.88) but not for Luminal B (Her2-positive). It was related to an even lower risk of mortality for $\mathrm{TNBC}(\mathrm{HR}=0.89,95 \% \mathrm{CI} 0.31-2.55)$. Furthermore, in the Luminal A subgroup, MetS had a significant effect on the mortality of patients with post-menopausal status, normal BMI, and Stage III BC. However, each component of MetS was not related to the risk of mortality in each molecular subtype, except for the Luminal B (Her2-positive) subgroup, in which high cholesterol was significantly associated with mortality $(\mathrm{HR}=1.93 .95 \% \mathrm{CI} 1.01-3.68)$. 


\section{Discussion}

In the present cohort study, MetS had an independent impression on the prognosis of BC, especially among the subgroups of Luminal A and Luminal B (Her2-negative), in which over a threefold increase in the risk of mortality was observed. These findings were in strong agreement with the data obtained from other studies, which showed that MetS had a 2.12-fold greater risk of mortality (in a US study) and 2.17-fold higher risk of relapse (in an Italian study) $(9,11)$. Given the heterogeneity of BC, clinical characteristics of the tumor were not sufficiently precise to evaluate the prognosis of early BC (18). Therefore, the molecular subtypes of BC are important as guides in optimal management during the long follow-up period. Some studies supported the great relationship between MetS and poor BC prognosis based on the outcomes linking MetS with advanced cancer stages, relapse, and mortality $(9,11)$. Watanabe reported that the multivariate-adjusted HR of death from BC was $11.9(95 \%$ CI 2.25-62.84) in Japanese women (19). The data were much higher than our data on BC mortality $(\mathrm{HR}=2.06)$ but were similar to our subgroup findings in advanced stage BC with Luminal A subtype ( $\mathrm{HR}=13.4)$.

Some studies indicated that obesity was an important prognosis factor related to an increased risk of mortality $(20,21)$. In the Asia-Pacific region, being overweight and/or obese was significantly 1.63 -fold related to the greater risk of mortality from BC. Low HDL-C was an important risk indicator for the prognostic effect on BC women with MetS, which was consistent with our findings (9). Even after analyzing the relationship between the prognosis and other components of MetS, including total 
cholesterol, triglycerides, and HDL, the conclusion was still uncertain $(11,14,22)$.

The previous findings that the prognostic effect of MetS was analyzed according to the molecular subtypes were not in exact agreement. In the Crosstalk between the ER and IGF pathways, obesity may promote ER-positive BC progression and endocrine therapy resistance, because obesity and the related increase in adiposity could intensify the metabolic pathways involved in aromatase regulation, which converts androgens to estrogen $(23,22)$. Goodwin found that a high level of serum insulin could impact the mortality of ER-negative cases (24). Dibaba and colleagues reported that in the subgroup of ER-positive BC, the presence of MetS increased the risk of mortality by $28 \%$, whereas the risk was lowered by $56 \%$ in the ER-negative subgroup. Moreover, missing Her2 status is one of the primary reasons affecting the prediction of the mortality of BC subtypes (11). Hypercholesterolemia is another factor that may influence the prognosis of HR-positive post-menopausal patients with BC. Longer duration of aromatase inhibitor use has more than twice the risk of contracting hypercholesterolemia compared with tamoxifen (25). Our research further confirmed that MetS was significantly associated with the mortality in the HRpositive Her2-negative subgroup, especially in Luminal A. Additionally, the subgroup patients of HR-positive Her2-negative may experience MetS due to the impact of long-term use of tamoxifen or aromatase inhibitor. Although we did not have data regarding the subjects' treatment history, long-term medication therapy can lead to MetS or its complications.

MetS influences the risk and mortality of $\mathrm{BC}$ through some potential biological 
pathways. Insulin can encourage the proliferation of some BC cells, and hyperinsulinemia is an independent risk factor of BC. Furthermore, blocking the IGF pathway may be an effective method for cases of hormone therapy-resistant BC (2628). In Her2-positive patients with $\mathrm{BC}$ who received neo-chemotherapy, the low level of IGF-1 was significantly associated with higher $\mathrm{pCR}$ rate but not with recurrence and mortality (29).

MetS is now recognized as a reversible condition depending on the degree of intervention. Physical activity and specific dietary patterns appeared to provide improved protection. Some trials have consistently reported that adherence to the Mediterranean diet is linked with the reduced risk of $\mathrm{BC}$ and is believed as protective factor in the progress of $\mathrm{BC}(30,31)$. In terms of the association between Mediterranean diet and molecular subtypes of $\mathrm{BC}$, the recent systematic review indicated that the Mediterranean diet pattern was negatively associated with $\mathrm{BC}$ risk particularly in ER- tumors subgroup and ER-/PR-/HER2-("triple-negative" tumors) (32). Among ER- tumors, the influence of dietary factors may be greater and easier to detect than among ER+ tumors because of the strong influence of hormonal factors $(33,34)$.

Admittedly, the analysis of the abovementioned results has some limitations. First, the analysis was based on the retrospective cross-sectional single-center design with a relatively modest number of MetS patients $(\mathrm{N}=199)$. Second, the National Cholesterol Education Program-Adult Treatment Panel criteria and the International Diabetes Federation standards have been widely used to define MetS. The range of 
the diagnostic agreement of MetS was from $75 \%$ to $88 \%$ when compared by pairing with other criteria (35). The different definitions of MetS may lead to its inconsistent prognostic assessment $(36,37)$. Third, all the patients with $\mathrm{BC}$ were recruited between 2010 and 2014. During six years, an update on the therapeutic options for BC might potentially lead to judgment bias.

\section{Conclusions}

In summary, MetS was an independent risk factor of mortality with $\mathrm{BC}$, especially in the subset of Luminal A. Additional larger prospective findings are required to better explain the effect of MetS on the molecular subtypes of BC.

\section{List of abbreviations}

MetS: Metabolic syndrome; BC: Breast cancer; HR: Hormone-receptor; CMADS:

Chinese Medical Association Diabetes Society; BMI: Body mass index; ER: Estrogen receptor; PR: Progestogen receptor; TNBC: Triple-negative breast cancer; DFS:

Disease-free survival; HR: Hazard ratio; CI: Confidence interval;

\section{Declarations}

\section{Ethics approval and consent to participate}

This study was approved by the Ethics Committee of the First Affiliated Hospital of Wenzhou Medical University. Written informed consent was required for all patients included in the study. No animal was involved in this study. 


\section{Consent for publication}

Not applicable.

\section{Availability of data and materials}

All data are available within the article.

\section{Competing interests}

The authors declare that they have no competing interests.

\section{Funding}

This work was supported by grants from Zhejiang Provincial Natural Science

Foundation of China under Grant No. LGF18H160031. Scientific Research

Foundation of Wenzhou, Zhejiang Province, China (Y20170740)

\section{Authors' contributions}

Jie You and Yefeng Cai designed the study and wrote the manuscript. Yuying Zhou, Endong Chen, and Dishuang Hu collected data. Jie You performed the statistical analyses. Xuan Shao prepared the figures. All authors have made an intellectual contribution to the manuscript and approved the submission.

\section{Acknowledgements}

Not applicable. 


\section{References}

1. Bray F, Ferlay J, Soerjomataram I, Siegel RL, Torre LA, Jemal A. Global cancer statistics 2018: GLOBOCAN estimates of incidence and mortality worldwide for 36 cancers in 185 countries. CA: a cancer journal for clinicians. 2018;68(6):394-424.

2. Sung H, Rosenberg PS, Chen WQ, Hartman M, Lim WY, Chia KS, et al. The impact of breast cancer-specific birth cohort effects among younger and older Chinese populations. International journal of cancer. 2016;139(3):527-34.

3. Sung H, Rosenberg PS, Chen WQ, Hartman M, Lim WY, Chia KS, et al. Female breast cancer incidence among Asian and Western populations: more similar than expected. Journal of the National Cancer Institute. 2015;107(7).

4. Zeng H, Chen W, Zheng R, Zhang S, Ji JS, Zou X, et al. Changing cancer survival in China during 2003-15: a pooled analysis of 17 population-based cancer registries. The Lancet Global health. 2018;6(5):e555-e67.

5. Allemani C, Matsuda T, Di Carlo V, Harewood R, Matz M, Niksic M, et al. Global surveillance of trends in cancer survival 2000-14 (CONCORD-3): analysis of individual records for 37513025 patients diagnosed with one of 18 cancers from 322 population-based registries in 71 countries. Lancet. 2018;391(10125):1023-75.

6. Runowicz CD, Leach CR, Henry NL, Henry KS, Mackey HT, Cowens-Alvarado RL, et al. American Cancer Society/American Society of Clinical Oncology Breast Cancer Survivorship Care Guideline. CA: a cancer journal for clinicians. 2016;66(1):43-73.

7. Grundy SM, Cleeman JI, Daniels SR, Donato KA, Eckel RH, Franklin BA, et al. Diagnosis and management of the metabolic syndrome: an American Heart Association/National Heart, Lung, and Blood Institute scientific statement: Executive Summary. Critical pathways in cardiology. 2005;4(4):198203.

8. Esposito K, Chiodini P, Colao A, Lenzi A, Giugliano D. Metabolic syndrome and risk of cancer: a systematic review and meta-analysis. Diabetes care. 2012;35(11):2402-11.

9. Berrino F, Villarini A, Traina A, Bonanni B, Panico S, Mano MP, et al. Metabolic syndrome and breast cancer prognosis. Breast cancer research and treatment. 2014;147(1):159-65.

10. Calip GS, Malone KE, Gralow JR, Stergachis A, Hubbard RA, Boudreau DM. Metabolic syndrome and outcomes following early-stage breast cancer. Breast cancer research and treatment. 2014;148(2):363-77.

11. Dibaba DT, Ogunsina K, Braithwaite D, Akinyemiju T. Metabolic syndrome and risk of breast cancer mortality by menopause, obesity, and subtype. Breast cancer research and treatment. 2019;174(1):209-18.

12. Grybach SM, Polishchuk LZ, Chekhun VF. Analysis of the survival of patients with breast cancer depending on age, molecular subtype of tumor and metabolic syndrome. Experimental oncology. 2018;40(3):243-8.

13. Agresti R, Meneghini E, Baili P, Minicozzi P, Turco A, Cavallo I, et al. Association of adiposity, dysmetabolisms, and inflammation with aggressive breast cancer subtypes: a cross-sectional study. Breast cancer research and treatment. 2016;157(1):179-89.

14. Lofterod T, Mortensen ES, Nalwoga H, Wilsgaard T, Frydenberg H, Risberg T, et al. Impact of prediagnostic triglycerides and HDL-cholesterol on breast cancer recurrence and survival by breast cancer subtypes. BMC Cancer. 2018;18(1):654. 
15. Chinese Metabolic Syndrome Study Cooperation. Suggestions about metabolic syndrome of Chinese diabetes society. Chin J Diab. 2004;12:156-61.

16. Edge SB, Compton CC. The American Joint Committee on Cancer: the 7th edition of the AJCC cancer staging manual and the future of TNM. Annals of surgical oncology. 2010;17(6):1471-4.

17. Goldhirsch A, Winer EP, Coates AS, Gelber RD, Piccart-Gebhart M, Thurlimann B, et al. Personalizing the treatment of women with early breast cancer: highlights of the St Gallen International Expert Consensus on the Primary Therapy of Early Breast Cancer 2013. Annals of oncology : official journal of the European Society for Medical Oncology. 2013;24(9):2206-23.

18. Weiss A, Chavez-MacGregor M, Lichtensztajn DY, Yi M, Tadros A, Hortobagyi GN, et al. Validation Study of the American Joint Committee on Cancer Eighth Edition Prognostic Stage Compared With the Anatomic Stage in Breast Cancer. JAMA oncology. 2018;4(2):203-9.

19. Watanabe J, Kakehi E, Kotani K, Kayaba K, Nakamura Y, Ishikawa S. Metabolic syndrome is a risk factor for cancer mortality in the general Japanese population: the Jichi Medical School Cohort Study. Diabetology \& metabolic syndrome. 2019;11:3.

20. Connor AE, Baumgartner RN, Pinkston C, Baumgartner KB. Obesity and risk of breast cancer mortality in Hispanic and Non-Hispanic white women: the New Mexico Women's Health Study. J Womens Health (Larchmt). 2013;22(4):368-77.

21. Nichols HB, Trentham-Dietz A, Egan KM, Titus-Ernstoff L, Holmes MD, Bersch AJ, et al. Body mass index before and after breast cancer diagnosis: associations with all-cause, breast cancer, and cardiovascular disease mortality. Cancer epidemiology, biomarkers \& prevention : a publication of the American Association for Cancer Research, cosponsored by the American Society of Preventive Oncology. 2009;18(5):1403-9.

22. Gerard C, Brown KA. Obesity and breast cancer - Role of estrogens and the molecular underpinnings of aromatase regulation in breast adipose tissue. Molecular and cellular endocrinology. 2018;466:15-30.

23. Subbaramaiah K, Howe LR, Bhardwaj P, Du B, Gravaghi C, Yantiss RK, et al. Obesity is associated with inflammation and elevated aromatase expression in the mouse mammary gland. Cancer Prev Res (Phila). 2011;4(3):329-46.

24. Goodwin PJ, Ennis M, Bahl M, Fantus IG, Pritchard KI, Trudeau ME, et al. High insulin levels in newly diagnosed breast cancer patients reflect underlying insulin resistance and are associated with components of the insulin resistance syndrome. Breast cancer research and treatment. 2009;114(3):51725.

25. Amir E, Seruga B, Niraula S, Carlsson L, Ocana A. Toxicity of adjuvant endocrine therapy in postmenopausal breast cancer patients: a systematic review and meta-analysis. Journal of the National Cancer Institute. 2011;103(17):1299-309.

26. Gunter MJ, Hoover DR, Yu H, Wassertheil-Smoller S, Rohan TE, Manson JE, et al. Insulin, insulinlike growth factor-I, and risk of breast cancer in postmenopausal women. Journal of the National Cancer Institute. 2009;101(1):48-60.

27. Rose DP, Vona-Davis L. The cellular and molecular mechanisms by which insulin influences breast cancer risk and progression. Endocrine-related cancer. 2012;19(6):R225-41.

28. Iida M, Tsuboi K, Niwa T, Ishida T, Hayashi SI. Compensatory role of insulin-like growth factor 1 receptor in estrogen receptor signaling pathway and possible therapeutic target for hormone therapyresistant breast cancer. Breast Cancer. 2019;26(3):272-81.

29. Tong YW, Wang G, Wu JY, Huang O, He JR, Zhu L, et al. Insulin-like growth factor-1, metabolic 
abnormalities, and pathological complete remission rate in HER2-positive breast cancer patients receiving neoadjuvant therapy. OncoTargets and therapy. 2019;12:3977-89.

30. Toledo E, Salas-Salvado J, Donat-Vargas C, Buil-Cosiales P, Estruch R, Ros E, et al. Mediterranean Diet and Invasive Breast Cancer Risk Among Women at High Cardiovascular Risk in the PREDIMED Trial: A Randomized Clinical Trial. JAMA internal medicine. 2015;175(11):1752-60.

31. Zuniga KE, Parma DL, Munoz E, Spaniol M, Wargovich M, Ramirez AG. Dietary intervention among breast cancer survivors increased adherence to a Mediterranean-style, anti-inflammatory dietary pattern: the Rx for Better Breast Health Randomized Controlled Trial. Breast cancer research and treatment. 2019;173(1):145-54.

32. Coughlin SS, Stewart J, Williams LB. A review of adherence to the Mediterranean diet and breast cancer risk according to estrogen- and progesterone-receptor status and HER2 oncogene expression. Annals of epidemiology and public health. 2018;1.

33. Fung TT, Hu FB, McCullough ML, Newby PK, Willett WC, Holmes MD. Diet quality is associated with the risk of estrogen receptor-negative breast cancer in postmenopausal women. The Journal of nutrition. 2006;136(2):466-72.

34. Bonanni AE, Bonaccio M, di Castelnuovo A, de Lucia F, Costanzo S, Persichillo M, et al. Food labels use is associated with higher adherence to Mediterranean diet: results from the Moli-sani study. Nutrients. 2013;5(11):4364-79.

35. Association C. The Suggestion on Chinese Metabolic Syndrome.Shanghai, China: Chinese Medical Association. 2004.

36. Tan CE, Ma S, Wai D, Chew SK, Tai ES. Can we apply the National Cholesterol Education Program Adult Treatment Panel definition of the metabolic syndrome to Asians? Diabetes care. 2004;27(5):11826.

37. Xi B, He D, Hu Y, Zhou D. Prevalence of metabolic syndrome and its influencing factors among the Chinese adults: the China Health and Nutrition Survey in 2009. Preventive medicine. 2013;57(6):867-71. 


\section{FIGURES}

Figure 1. Kaplan-Meier survival curves showing DFS (A) and OS (B) in BC patients stratified by MetS.

\section{TABLES}

Table 1. Baseline characteristics of breast cancer patients stratified by mets.

Table 2. Cox proportional hazards regression models of risk factors associated with prognosis among $\mathrm{BC}$ patients.

Table 3. Cox proportional hazard regression analysis of overall and disease-free survival in BC adjusting for MetS

Table 4. Cox regression analysis of OS in the molecular subgroups of BC patients. 
Figures
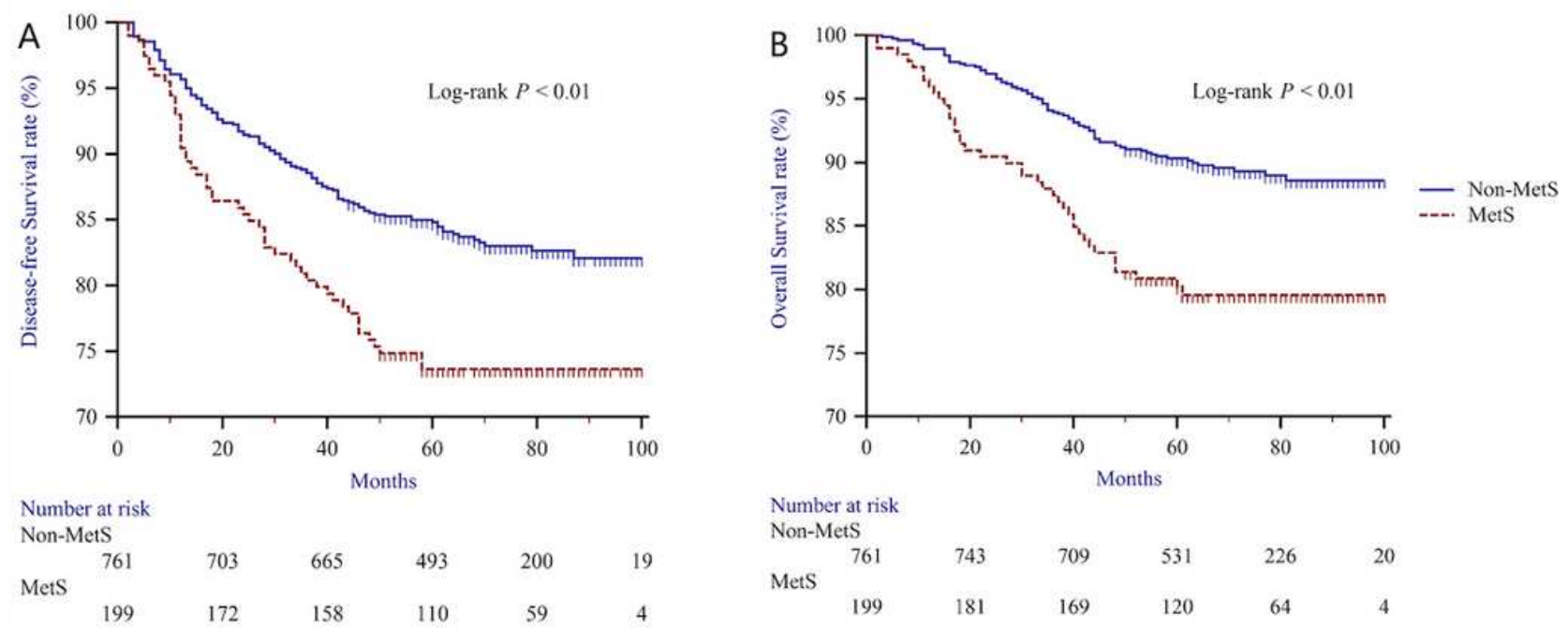

\begin{tabular}{|c|c|c|c|c|c|c|}
\hline $\begin{array}{l}\text { Numb } \\
\text { Non- }\end{array}$ & $\begin{array}{l}\text { at ris } \\
\text { tS }\end{array}$ & & & & & \\
\hline & 761 & 743 & 709 & 531 & 226 & 20 \\
\hline & 199 & 181 & 169 & 120 & 64 & 4 \\
\hline
\end{tabular}

\section{Figure 1}

Kaplan-Meier survival curves showing DFS (A) and OS (B) in BC patients stratified by MetS. 